\title{
Problemas dogmáticos RELACIONADOS CON EL DELITO DE VIOLACIÓN CON HOMICIDIO: COMENTARIO A LA SENTENCIA RIT 115-2015, DEL TRIBUNAL ORAL EN LO PENAL DE LA SERENA*
}

\section{JAVIER ESCOBAR VEAS ${ }^{* *}$}

\section{1) INTRODUCCIÓN}

El presente comentario versa sobre la sentencia definitiva emitida por el Tribunal Oral en lo Penal de La Serena (en adelante el Tribunal), en causa RIT 115-2015', que condenó al acusado de iniciales R.L.A.S. (en adelante el imputado) por el delito de violación, en concurso real con el de homicidio simple, a sendas penas de ochocientos dieciocho días de presidio menor en su grado medio y quince años de presidio mayor en su grado medio, respectivamente, descartando la aplicación del delito de violación con homicidio, pretendida por el Ministerio Público.

La sentencia que a continuación se comentará se ha escogido en atención a los problemas dogmáticos que ella enfrenta y resuelve. En efecto, con prescindencia del mérito de las conclusiones jurídicas a las que arriba el Tribunal, debe valorarse positivamente el hecho de que identifica tópicos problemáticos y los aborda dogmáticamente, citando doctrina pertinente y exponiendo argumentos en pos de su postura.

La estructura del comentario se dividirá de la siguiente forma.

En el primer apartado se transcribirán tanto los hechos que se tuvieron por acreditados por el Tribunal como aquellos que formaron parte de la acusación fiscal. Además, se señalará la calificación jurídica otorgada por el Ministerio Público a su acusación, y la calificación jurídica dada por el Tribunal a los hechos acreditados, explicitando sus diferencias.

Luego, en los posteriores apartados, se expondrán y revisarán críticamente aquellos tópicos que denominamos problemas dogmáticos, y que fueron tratados a su vez por el Tribunal, a saber: el significado de la expresión "acceso carnal"; el iter criminis en el delito de violación con homici-

\footnotetext{
* Fecha de recepción: 16 de octubre de 2016. Fecha de aceptación: 12 de diciembre de 2016.

** Abogado, Magíster en Derecho Penal y Procesal Penal Universidad Diego Portales, Profesor de Derecho Penal e Investigador del Centro de Estudios Penales de la Universidad Mayor.

1 Tribunal de Juicio Oral en lo Penal de la Serena. 12 de mayo de 2015. RIT 115-2015. Disponible en: http://www.pjud.cl/documents/396729/0/ Sentencia+TOP+La+Serena+-+homicidio+Vicuna.pdf/a46d6981-5b51-434d-8039-e4aac9bbbb6f [fecha de visita: 24 de octubre de 2016], 44 pp.
} 
$\mathrm{dio}^{2}$; el sentido de la expresión "con ocasión”, y finalmente, el problema del concurso aparente entre el delito de violación en estado de ejecución imperfecto y el delito de abuso sexual en grado de consumación.

\section{2) Hechos acreditados y alegaciones del Ministerio Público}

Como se señaló precedentemente, en este apartado se indicarán los hechos que el Tribunal tuvo por acreditados, y aquellos contenidos en la acusación de la Fiscalía.

En relación a los hechos probados, estos son los siguientes:

"Cuarto: Que a través de la prueba pericial, gráfica, documental y testimonial, se acreditó que el día 27 de abril de 2014, pasada las 06:00 horas de la madrugada, C.V.H. ${ }^{3}$, fue acometida en las cercanías de una acequia, inmediata a la calle San Martín, frente al $\mathrm{n}^{\circ}$ 628 de la localidad de Vicuña, por un sujeto que mediante diversas agresiones levantó su ropa superior por sobre sus pechos y le bajó el pantalón y calzón hasta las proximidades de las rodillas, para accederla carnalmente con su pene, causándole erosiones y equimosis en la zona perihimenal, pero sin penetrarla en la vagina, sujeto que, continuando con su actividad agresiva, la golpeó en la cabeza y aprovechando, además, el evidente estado de embriaguez en que se encontraba la ofendida, la arrastró hasta una poza de agua contigua, arrojándola a ella, lo que le provocó la muerte por asfixia por inmersión”.

Respecto de los hechos materia de la acusación fiscal, estos fueron:

"El 27 de abril de 2014, aproximadamente entre las 05:50 horas y las 09:10 horas, en un potrero ubicado en calle San Martín Norte, frente a la casa signada con el número 628, Vicuña, el acusado, con la finalidad de acceder carnalmente a la afectada C.A.V.H. y vencer la oposición de ésta, procedió a agredirla en diferentes partes del cuerpo con elemento contuso, golpes de puño y rasguńos, procediendo luego a introducir su pene en la vagina de la víctima, a quien arrastró hasta a un canal de regadío existente en el lugar, donde finalmente falleció debido a asfixia por sumersión (...)".

2 Delito contemplado en el artículo 372 bis del Código Penal, cuyo tenor es el siguiente: "Artículo 372 bis.- El que, con ocasión de violación, cometiere además homicidio en la persona de la víctima, será castigado con presidio perpetuo a presidio perpetuo calificado". Desde ahora en adelante, cuando se señale algún artículo, sin expresar cuerpo legal, deberá entenderse que nos estamos refiriendo al Código Penal nacional.

3 Se ha decidido reemplazar los nombres de la víctima y del imputado por las iniciales de cada uno de ellos, en atención a la naturaleza de los delitos imputados. 
Como se puede apreciar, existió una importante diferencia entre los hechos materia de la acusación y aquellas circunstancias fácticas que se tuvieron por acreditadas durante el juicio. En efecto, mientras que en la acusación el Ministerio Público señaló que el imputado habría introducido su pene en la vagina de la víctima, el Tribunal tuvo por acreditado que no habría existido tal penetración.

Consecuente con su propuesta fáctica, la Fiscalía calificó jurídicamente los hechos de la acusación como un delito de violación con homicidio, del artículo 372 bis, en calidad de autor y en grado de desarrollo consumado. El Tribunal, por las razones que se comentarán más adelante, calificó jurídicamente los hechos acreditados como un concurso real entre un delito de violación tentada, y un delito de homicidio consumado, ambos en calidad de autor.

Si bien la diferencia fáctica, en términos cuantitativos, puede resultar menor, no puede sino afirmarse que en términos jurídicos dicha disparidad acarreó consecuencias de relevancia, pasando de la calificación como delito complejo (violación con homicidio) a un concurso entre dos figuras básicas (violación y homicidio), además de verse modificado el grado de desarrollo del delito.

A continuación se abordarán los problemas dogmáticos que debió enfrentar y resolver el Tribunal.

\section{3) SigNIFICADO DE LA EXPRESIÓN “ACCESO CARNAL”}

El artículo 361 señala que comete violación el que accede carnalmente, por vía vaginal, anal o bucal, a una persona, siempre que concurra alguna de las circunstancias allí enunciadas.

Se ha entendido unánimemente que la acción típica incriminada en el ilícito mencionado consiste en acceder carnalmente a otro. Si entendemos que la consumación se alcanza cuando se cumplen todos los elementos del tipo penal ${ }^{4}$, el acceso carnal fundamentaría precisamente una atribución delictiva a título de consumación, toda vez que con dicho acceso el tipo penal estaría completo.

Nuestra doctrina ha entendido mayoritariamente la expresión "acceso carnal" como la introducción del miembro reproductor masculino, con

Muñoz, Juan (1992) El delito de detención. Madrid: Trotta, 312 pp., p. 141; Mañalich, Juan Pablo (2004) "El secuestro como delito permanente frente al DL de Amnistías". Revista de Estudios de la Justicia, $\mathrm{N}^{\circ}$ 5, pp. 11-33. Disponible en: http://web.derecho.uchile.cl/ cej/recej/recej4/archivos/tentativa\%20y\%20desistimiento\%20_JPM_corregido\%20_16_. pdf [fecha de visita: 14 de septiembre de 2016], p. 3. 
exclusión de objetos, extremidades o prolongaciones corporales (lengua, dedos $)^{5}$.

La sentencia comentada incurre, en este punto, en un error. En efecto, en su Considerando Cuarto, al expresar los hechos que se tuvieron por acreditados, señala: "para accederla carnalmente con su pene, causándole erosiones y equimosis en la zona perihimenal, pero sin penetrarla en la vagina”.

Como se puede apreciar, el Tribunal afirma que existió acceso carnal, sin embargo luego señala que no hubo penetración vaginal. Lo anterior evidencia un erróneo concepto sobre la expresión "acceso carnal", toda vez que según el Tribunal este puede existir sin penetración, contradiciendo la opinión mayoritaria de la doctrina. El significado de la expresión acceso carnal requiere la introducción (penetración) del miembro reproductor masculino, tal como lo han entendido nuestros autores ${ }^{6}$.

\section{4) ITER CRIMINIS EN EL DELITO DE VIOLACIÓN CON HOMICIDIO}

Probablemente el problema dogmático de mayor relevancia tratado en la sentencia sea la aplicación de las normas sobre iter criminis en el delito de violación con homicidio ${ }^{7}$.

Lo primero que debemos seńalar es que este tópico indudablemente forma parte de uno más amplio, a saber, la aplicación de las normas de iter criminis en los delitos complejos. Sin embargo, el examen de este excedería con creces los límites del presente trabajo.

Primero que todo, es imprescindible destacar que la mayoría de los autores que se citarán en este apartado y en el siguiente, efectúan sus reflexiones a propósito del delito de robo con homicidio, y no específi-

5 Carrasco, Edison (2007) "El problema del sujeto activo del delito de violación y sus posibles vacíos legales". Revista Ius et Praxis, Vol. 13, N² 2, pp. 137-155. Disponible en: http:// www.scielo.cl/pdf/iusetp/v13n2/art07.pdf [fecha de visita: 19 de abril de 2016], pp. 3 y 4; Politoff, Sergio; Matus, Jean Pierre; y Ramírez María Cecilia (2006) Lecciones de Derecho Penal Chileno, Parte Especial, 2a Edición. Santiago: Editorial Jurídica de Chile, 691 pp., p. 251; Bullemore, Vivian; Mackinnon, John, (2005) Curso de Derecho Penal. Santiago: Editorial LexisNexis, T. III, 261 pp., p. 186; Rodríguez, Luis (2015) Delitos Sexuales. Santiago: Editorial Jurídica de Chile, 458 pp., pp. 181 y 182; Garrido, Mario (2010) Derecho Penal, Parte Especial. Santiago: Editorial Jurídica de Chile, T. III, 515 pp., pp. 272 y 273; Balmaceda, Gustavo (2014) Manual de Derecho Penal, Parte Especial. Santiago: Editorial Librotecnia, 731 pp., p. 216.

6 Ver nota número 5.

7 Desde ya cabe hacer presente que este tópico forma parte de uno más vasto, a saber, la compatibilidad o no, y la forma concreta en que ella tendría lugar, de las reglas de iter criminis en los delitos complejos, y en particular en algunas hipótesis complejas calificadas. Debido al objeto y a la estructura del presente trabajo, un análisis general sobre el asunto no es posible en esta oportunidad. 
camente a la luz del delito de violación con homicidio ${ }^{8}$. Tras analizar sus reflexiones, estimamos que no existen obstáculos para extrapolar sus conclusiones desde aquel delito hasta este, ello por cuanto sus argumentos no se elaboran en base a alguna característica exclusiva del tipo penal de robo con homicidio, sino que se esgrimen sobre elementos que pueden ser encontrados también en el delito de violación con homicidio.

Sobre el punto a dilucidar, entonces, podemos señalar que en nuestra doctrina existen dos posturas al respecto: una que admite sin problema alguno la aplicación de estas normas - nos referimos a las de iter criminis- y otra que la rechaza.

Como representantes de la primera posición podemos mencionar, en primer lugar, a Etcheberry. A propósito del delito de robo con homicidio, este autor sostiene que es perfectamente posible apreciar tentativa según las normas generales. Lo que se requeriría sería que el autor haya dado principio de ejecución a ambos delitos base, con el factor de imputación subjetiva requerido. Por tanto, cuando el agente no haya dado principio de ejecución al homicidio, sino que solamente al robo, no será posible apreciar tentativa de robo con homicidio ${ }^{9-10}$. En el mismo sentido se expresa Garrido, quien señala que "respecto de la consumación del delito y sus grados imperfectos, rigen los principios generales que se seńalaron" a propósito del delito de robo con violencia o intimidación, vale decir, se admite expresamente la posibilidad de apreciar las formas imperfectas de ejecución ${ }^{11}$. Jaime Vivanco y Politoff, Matus y Ramírez también aceptan la posibilidad de aplicar las normas de iter criminis al delito de robo con homicidio $^{12}$.

A su vez, como representantes de la segunda postura podemos citar a Luis Rodríguez y a Guillermo Oliver. Este último autor señala que "en relación con el castigo de las etapas anteriores a la consumación, los autores afirman que las disposiciones que permiten sancionar dichas etapas son aplicables al robo con homicidio (...) y que tiene aplicación respecto de esta figura lo dispuesto por el artículo 450". Este planteamiento, afirma

8 Esta situación puede deberse, principalmente, a dos cuestiones: En primer lugar, a la ausencia de monografías sobre el estudio del delito en cuestión; y en segundo lugar, a las similitudes que presentan ambos tipos penales. En efecto, ambas normas -tanto el art. 372 bis como el 433- utilizan la expresión "con ocasión", contemplan al homicidio como una de sus hipótesis, y responden a la estructura de aquellos delitos complejos calificados.

9 Etcheberry, Alfredo (1998) Derecho Penal. Santiago: Editorial Jurídica de Chile, T. III, 490 pp., p. 343.

10 Etcheberry afirma, además, que "debe recordarse (...) que, encontrándose ya este delito complejo en grado de tentativa, debe imponérsele la pena correspondiente al delito consumado". ETCHEBERry (1998) 343.

11 Garrido, Mario (2014) Derecho Penal, Parte Especial. Santiago: Editorial Jurídica de Chile, T. IV, 419 pp., pp. 202, 203 y 212.

12 Vivanco, Jaime (2009) El Delito de Robo con Homicidio. Santiago: Legal Publishing, 81 pp., pp. 57 a 60; y Politoff; Matus; y Ramírez (2006) 370. 
Oliver, "no solo es cuestionable en atención a los fundamentos que inspiran la norma recién aludida, sino también porque puede llevar a situaciones francamente inaceptables" 13 . El autor rechaza la aplicación de las normas sobre iter criminis al delito comentado, toda vez que "lo que un sector de la doctrina parece olvidar es que el artículo $433 \mathrm{~N}^{\circ} 1 \mathrm{CP}$ exige que tanto la apropiación como el homicidio se encuentren consumados". La conclusión anterior, para Oliver, fluye claramente del hecho de haberse utilizado el verbo cometer en conjunto con el vocablo además, lo cual implica que robo y homicidio deben estar sujetos a los mismos requisitos. Concluye Oliver que si alguno de los dos hechos no alcanza su consumación, no corresponde sancionar a título de robo con homicidio tentado o frustrado, sino aplicar las reglas generales concursales ${ }^{14-15}$.

¿Qué sostuvo el Tribunal a propósito de este tema? Recordemos que en el Considerado Quinto señaló:

"QUINTO: Que tales hechos configuran un delito tentado de Violación, en concurso material con un delito de Homicidio simple, en la persona de C.V.H., previstos y sancionados en el artículo $361 \mathrm{n}^{\circ} 1 \mathrm{e}$ inciso tercero del artículo $7^{\circ}$, ambos del Código Penal, y artículo 391 $\mathrm{n}^{\circ} 2$ del mismo cuerpo legal. (...).

Ahora bien, para efectuar la aplicación de la pena estatuida en el artículo 372 bis del Código Penal, ello presupone que tanto la violación como el homicidio se encuentren consumados, y ello se evidencia del empleo de la expresión "cometiere además homicidio", expresión que implica que tanto este último delito como la violación deben encontrarse cometidos, es decir, consumados, por lo que no cabría hablar de tentativa ni delito frustrado de violación con homicidio”.

Como se puede apreciar, el Tribunal adscribe a la segunda de las posturas reseñadas, acogiendo, sin citar expresamente, la tesis de Oliver y Rodríguez. En efecto, el Tribunal señala que el tipo penal, al utilizar la expresión "cometiere además homicidio", exige que tanto el homicidio como la violación estén en el estado de consumación, por lo que las normas de iter criminis no se aplicarían al tipo penal en estudio.

Según nuestra opinión, es posible arribar a la conclusión del Tribunal -y de la Doctrina a la cual ésta adscribe-; sin embargo, los argumentos

13 Oliver, Guillermo (2013) Delitos contra la Propiedad. Santiago: Thomson Reuters, 591 pp., p. 318.

$14 \quad$ Oliver (2013) 319.

15 Mismo argumento expone Rodríguez, Luis (2009) "Robo con Homicidio". Revista de Estudios de la Justicia, $\mathrm{N}^{\circ}$ 11, pp. 131-151. Disponible en: http://web.derecho.uchile.cl/cej/ rej11/RODRIGUEZ\%20COLLAO\%20_12_.pdf [fecha de visita: 10 de octubre de 2016], p. 144 . 
que utiliza son, a nuestro juicio, insuficientes para fundamentarla. En efecto, el argumento literal carece de fuerza suficiente para excluir la aplicación de las reglas sobre iter criminis de la figura en comento, toda vez que si se conciben los tipos tentados y frustrados como supeditados al tipo consumado, los primeros son en realidad homicidios, solo que en una etapa de ejecución anterior a la consumación. Por tanto, cuando la ley dice "homicidio", no excluye necesariamente el "homicidio tentado o frustrado". De esta forma, la voz homicidio sería un género que cobijaría los distintos estados de ejecución.

A mayor abundamiento, cabría agregar que al menos en una oportunidad nuestro legislador ha señalado expresamente el estado de ejecución relevante para efectos legales. A saber, el artículo $1^{\circ}$ de la Ley 18.216, que regula las penas sustitutivas a las penas privativas o restrictivas de libertad, excluye de dichas penas sustitutivas a los autores de los delitos en estado de consumación que a continuación menciona. Es decir, en este tópico nuestro legislador expresamente ha señalado que el delito debe estar en grado de consumación para efectos de excluir la procedencia de penas sustitutivas.

¿Significa lo anterior que la conclusión a que arriba el Tribunal es incorrecta? En nuestra opinión, la conclusión del Tribunal es posible, sin embargo el argumento que utiliza no es satisfactorio. Existen otras razones, relacionadas con la estructura y el fundamento de este tipo de delitos, que podrían permitir llegar a la misma afirmación ${ }^{16}$.

\section{5) SENTIDO DE LA EXPRESIÓN “CON OCASIÓN”}

Además de los razonamientos anteriormente expuestos, para descartar la aplicación del delito de violación con homicidio, el Tribunal añade otro en el Considerando Quinto, al expresar:

"De la disposición legal transcrita ${ }^{17}$, se evidencia que entre la violación y homicidio debe existir un vínculo, es decir, que el homicidio se cometa con ocasión de la violación, que la mayoría de la doctrina y la jurisprudencia considera que se trata de una proximidad o inmediatez temporal, pero esta proximidad en el tiempo entre la violación y el homicidio no es suficiente por sí misma, sino que es necesario que el homicidio ocurra con ocasión de actos que impliquen desde el punto de vista jurídico de ejecución o comienzo de ejecución de la violación”.

\footnotetext{
16 Lamentablemente por razones de estructura y extensión, no estamos en condiciones de plantear una tesis en este trabajo.

17 Se está refiriendo al artículo 372 bis. El texto de la norma fue transcrito en la nota número 1.
} 
A continuación analizaremos las opiniones dadas por la doctrina sobre el significado de la expresión "con ocasión", para luego analizar el razonamiento y la decisión del Tribunal.

Etcheberry señala a propósito del delito de robo con homicidio, que "cometerlo con ocasión (...) significa que el homicidio es para el delincuente una manera de lograr su seguridad o impunidad ${ }^{18}$; no es matar para robar, sino matar al robar. Supone, desde luego, la unidad de tiempo, circunstancias y lugar con el robo, pero además caracterizada por el hecho de que la forma y modo de comisión del robo permitan la ejecución de un homicidio que de no mediar aquellas circunstancias no habría sido posible ejecutar con la misma facilidad. Es decir, se aprovecha el debilitamiento de la defensa privada, la indefensión de la víctima, que ha resuelto de la violencia o intimidación propias del robo, para matar a aquélla. No exige (...) el designio anticipado de matar. Comprende todos los casos en que el homicidio es una consecuencia del robo, no un medio para cometerlo"19-20.

Según nuestra opinión, la expresión "con ocasión" no puede significar "para lograr su seguridad o impunidad", o al menos no puede significar necesariamente eso. Lo anterior se aprecia claramente al leer el artículo 432, donde se sanciona, entre otras hipótesis, la violación cometida con ocasión del robo. Si se entiende que "con ocasión" requiere que el agente obre con la intención de obtener la impunidad, no es posible imaginar cómo podría cometer un delito de violación para conseguir tal fin.

En base a las críticas anteriores, creemos que es preferible la definición de Garrido, quien señala que "la norma se aplica cuando el homicidio de la víctima se ha cometido con ocasión de la violación, esto es, dentro del contexto fáctico de su ejecución. Cuando el resultado muerte se encuentra descontextualizado fácticamente de la violación, se recurrirá a las reglas generales del concurso para sancionar esos hechos" 21 . Como se puede apreciar, este autor acentúa el elemento de temporalidad y espacialidad, acercándose al significado ordinario de la palabra "ocasión".

Ahora, volviendo al razonamiento del Tribunal, recordemos que este señala que "con ocasión" requiere de una proximidad o inmediatez tem-

En el mismo sentido Oliver (2013) 312, y RodríGuez (2009) 139.

ETCHEBERry (1998) 342.

Politoff, Matus y Ramírez, siguiendo a Etcheberry, sostienen que "si el hecho lo comete como una manera de asegurarse u obtener la impunidad, estaría actuando con ocasión del robo. Lo importante es que con la voz ocasión se destaca que no se requiere el designio anticipado (una suerte de premeditación) de matar cuando se ha planeado cometer el delito". Politoff; Matus; y Ramírez (2006) 369. Labatut señala que "con ocasión" significa que el homicidio se cometa a consecuencia del delito principal. Labatut, Gustavo (2000) Derecho Penal. Santiago: Editorial Jurídica de Chile, 263 pp., p. 203. 
poral -la cual sería necesaria, pero no suficiente-, y además que el homicidio ocurra con ocasión de actos que impliquen desde el punto de vista jurídico la ejecución o comienzo de ejecución de la violación. El Tribunal, por tanto, exige la concurrencia de dos elementos, a saber, proximidad temporal y que los actos que configuran el homicidio a su vez correspondan a actos de ejecución de la violación.

El primer elemento efectivamente es exigido por la doctrina, y estamos de acuerdo con él. De hecho, la expresión "con ocasión" exige indefectiblemente una proximidad temporal, es decir, que ambas hipótesis delictivas ocurran en un mismo contexto fáctico -según señala Garrido-. Sin embargo, el segundo elemento requerido por el Tribunal presenta grandes dificultades, y desde ya podemos señalar que a nuestro juicio es incorrecto.

Cuando el Tribunal exige que "el homicidio ocurra con ocasión de actos que impliquen desde el punto de vista jurídico de ejecución o comienzo de ejecución de la violación", restringe excesivamente el campo de aplicación del delito contenido en el artículo 372 bis, incluso contra el sentido claro de la ley. En efecto, debemos recordar que, si bien es posible imaginar que el homicidio ocurra con actos ejecutivos de la violación, no sucede lo mismo con algunas de las demás hipótesis donde nuestro legislador utiliza la expresión "con ocasión", siendo paradigmático el caso de la violación cometida con ocasión del robo (para que la tesis del Tribunal fuese correcta, el art. 433 exigiría que la violación se produjese por actos ejecutivos del delito de robo) ${ }^{22}$. Este segundo requisito, exigido por el Tribunal, pareciera consistir en una identidad de acción, estándar muy superior a lo que conocemos en otros ámbitos ${ }^{23}$.

Por consiguiente, en nuestra opinión el elemento "con ocasión" solamente exige la proximidad temporal y compartir un contexto fáctico determinado - de esta forma, se adscribe al concepto defendido por Garrido-.

Ahora bien, dicho lo anterior, cabe verificar si de conformidad con los hechos y la dinámica acreditados por el Tribunal, era posible apreciar la concurrencia del elemento "con ocasión", según lo señalado precedentemente.

El Tribunal tuvo por acreditado que, luego de intentar consumar la violación, surgió inmediatamente un ánimo de matar en el encausado. En dicho momento la víctima se encontraba diezmada producto de las

22 Otros ejemplos en que la tesis del Tribunal no podría tener aplicación, son los artículos 12 número 10, 141 inciso 5, 142 inciso 2, 266 inciso 2, y 368

23 A modo de ejemplo, se puede citar el artículo 75, que regula el concurso ideal. En esta hipótesis los autores son contestes en que no se exige identidad de acción. Por tanto, el estándar del Tribunal a propósito de este requisito sería incluso superior al del artículo 75 para apreciar concurso ideal. 
contusiones en la zona parietal, unido a su avanzado estado etílico. El imputado la arrojó enseguida a una poza con agua, donde su muerte por inmersión resultaba inminente, como finalmente ocurrió.

Así las cosas, creemos que en el caso comentado lo correcto hubiese sido apreciar la concurrencia del requisito "con ocasión", toda vez que el agente, luego de intentar consumar el delito de violación, inmediatamente toma a la víctima y la arroja a una poza con agua, provocando en definitiva su muerte ${ }^{24}$. El contexto fáctico es el mismo, e incluso existe una relación entre los dos momentos. Sin perjuicio de lo anterior, cabe recordar que, según lo expuesto en el apartado anterior, la aplicación del delito de violación con homicidio de todas maneras podría haberse descartado en virtud de las razones allí expuestas.

\section{6) CONCURSO APARENTE ENTRE EL DELITO DE VIOLACIÓN EN ESTADO DE EJECUCIÓN IMPERFECTO Y EL DELITO DE ABUSO SEXUAL EN GRADO DE CONSUMADO}

Mayoritariamente nuestra doctrina ha entendido que el delito de violación es de mera actividad, por tanto no es posible a su respecto la frustración como etapa de desarrollo del iter criminis ${ }^{25}$. Sin perjuicio de lo anterior, cabe reconocer que es posible encontrar sentencias en que nuestros Tribunales han admitido el estado de frustración en delitos de mera actividad, incluyendo, por cierto, el de violación ${ }^{26}$.

Recordemos que de conformidad con el artículo 52, a los autores de crimen o simple delito tentado se les debe imponer la pena asignada al delito, rebajada en dos grados. Siendo la pena legal asignada al delito de violación propia consumada la de presidio mayor en sus grados mínimo a medio, a los autores de violación tentada les corresponde la pena de presidio menor en su grado medio. Al mismo tiempo, la pena asignada al delito de abuso sexual propio consumado es de presidio menor en su grado máximo.

¿Cómo se debe resolver, entonces, un eventual concurso aparente entre los delitos de violación en estado de tentativa y de abuso sexual consumado?

24 Si bien las dos acciones (la de violación y la homicida) son claramente identificables, cabe recordar que "con ocasión" es más amplio que "con la misma acción".

25 Politoff; Matus; y Ramírez (2006) 260; Garrido (2010) T. III, 277; Rodríguez (2015) 211; Balmaceda (2014) 220. Como opinión minoritaria es posible señalar a Bullemore y Mackinnon, quienes afirman que "habrá que entenderse que el delito de violación, como cualquier otro, puede encontrarse en grado de tentativa, frustración o consumación”. BuLlemore; Mackinnon, (2005) 210.

26 Ramírez, María Cecilia (2005), "La frustración en delitos de mera actividad a la luz de determinadas sentencias". Revista de Derecho de la Pontificia Universidad Católica de Valparaíso, Vol. XXVI, pp. 133-141. Disponible en: http://www.rdpucv.cl/index.php/rderecho/article/ viewFile/570/538 [fecha de visita: 22 de agosto de 2016]. 
Tal como señalan Politoff, Matus y Ramírez, "el problema que surge no es tanto la existencia conceptual de la tentativa de violación, sino los efectos penológicos de su admisión" 27 . En efecto, la admisión de la tentativa de violación está fuera de discusión, sino que lo que debe resolverse es cómo calificar un hecho que configura, al mismo tiempo, el ilícito indicado recientemente y el de abuso sexual. Garrido señala que "no es fácil distinguir entre la comisión tentada de los delitos de violación o de estupro, del delito de abusos sexuales, particularmente cuando la manifestación objetiva de la conducta parece satisfacer claramente las exigencias de punibilidad de ambas figuras; sin embargo, han de recibir una sanción diversa según cuál sea dicha calificación" 28 .

Garrido concluye, a continuación, que en un caso donde se configura un delito de abuso sexual consumado, y los mismos hechos califiquen a su vez como violación tentada, se deberá sancionar en definitiva por el primer delito. Ello en virtud de una razón penológica (el primer delito tendría mayor pena que el segundo), pero además de conformidad con el principio de consunción, toda vez que "la ejecución tentada del delito de violación (o estupro) importa la puesta en peligro del bien jurídico, mientras que la ejecución consumada de los abusos sexuales, su lesión. En tanto coexiste el mismo interés jurídico protegidos en las dos figuras, resulta superior el disvalor delictivo del abuso sexual, que abarca la ejecución tenada de violación o estupro"29. En el mismo sentido Politoff, Matus y Ramírez, quienes afirman que ordinariamente la violación absorberá las demás agresiones de índole sexuales, entre las que se encuentran evidentemente los abusos sexuales. Sin embargo, "si la violación está en grado de tentativa la solución no es la misma”. En este caso el abuso sexual consumado ya no se encuentra copenado, sino que en virtud del principio de alternatividad deberá primar la pena más grave en concreto, para no castigar dos veces el mismo hecho ni conceder un absurdo privilegio, lo cual se traduce que en esta específica hipótesis se deberá sancionar por el delito de abuso sexual ${ }^{30}$.

Volviendo a la sentencia comentada, recordemos que el Tribunal condena al imputado por "un delito tentado de Violación, en concurso material con un delito de Homicidio simple". Consecuentemente, se impone, por el delito de violación tentada, la pena de ochocientos dieciocho días de presidio menor en su grado medio. Como se puede apreciar, el Tribunal rebajó en dos grados la pena asignada al delito.

¿Es correcta la solución del sentenciador? Para responder a la pregunta, primeramente se debe dilucidar si los hechos que se tuvieron

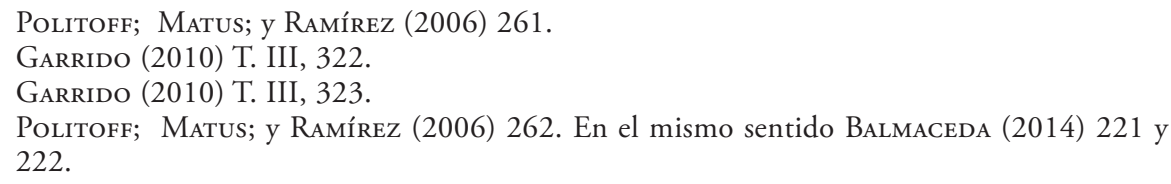


por acreditados pueden configurar o no un delito de abuso sexual, según lo dispuesto en los artículos 366, 366 bis y 366 ter. En lo pertinente, estos hechos fueron que el acusado, mediante diversas agresiones, "levantó la ropa superior de la víctima, le bajó el pantalón y calzón hasta las proximidades de las rodillas, y la accedió carnalmente con su pene ${ }^{31}$, causándole erosiones y equimosis en la zona perihimenal, pero sin penetrarla en la vagina”.

Como se puede apreciar, los hechos acreditados configurarían, sin mayor problema, un delito de abuso sexual del artículo 366. En efecto, la conducta del imputado indudablemente es de significación sexual y de relevancia, y concurre la circunstancia del número 1 del artículo $361^{32}$.

En este contexto, el Tribunal se vio enfrentado a la disyuntiva de qué tipo penal aplicar en relación a la conducta de significación sexual desplegada por el imputado. Lamentablemente, no existe constancia en la sentencia que este punto haya sido objeto de discusión durante el desarrollo del juicio $^{33}$. El Tribunal afirma, sin analizar las consecuencias del asunto, en el Considerando Quinto, que los hechos acreditados "configuran un delito tentado de Violación, en concurso material con un delito de Homicidio simple".

Así las cosas, no existiendo un razonamiento por parte del Tribunal en relación a la correcta calificación jurídica ni a sus consecuencias penológicas, no puede sino apreciarse un vacío argumentativo en la sentencia comentada. El asunto no es baladí, al menos no para el Ministerio Público, toda vez que en caso de haberse calificado el hecho como un delito de abuso sexual consumado, la pena por esta conducta podría haber llegado hasta los 5 años de presidio menor en su grado máximo.

\section{7) CONCLUSIONES}

La sentencia comentada enfrenta cuestiones dogmáticas de relevancia, y les asigna una respuesta que el Tribunal considera correcta, lo cual debe ser valorado positivamente.

Ahora bien, analizando directamente los tópicos dogmáticos resueltos por la sentencia, es posible identificar que estos fueron cuatro, a saber: significado de la expresión "acceso carnal", la aplicación o no de las reglas sobre iter criminis al delito de violación con homicidio, el sentido de la

Sobre este punto, ver supra 3).

El análisis de los requisitos de "significación sexual y de relevancia" superaría con creces el ámbito de este comentario. Sin embargo, nos parece que difícilmente podría cuestionarse su concurrencia en el caso concreto.

33 Recordemos que el Ministerio Público dedujo acusación por el delito de violación con homicidio, por tanto puede asumirse que este asunto no haya estado en su "órbita" de discusión. 
expresión "con ocasión", y finalmente las consecuencias de un eventual concurso aparente entre el delito de violación en estado de ejecución imperfecto y un delito de abuso sexual en estado de consumación.

En relación a la expresión "acceso carnal", el Tribunal yerra, a nuestro juicio, en su interpretación, al afirmar que en el caso de autos existió acceso carnal sin penetración. Concordamos con la opinión doctrinaria dominante, en el sentido de que el momento consumativo de la violación está dado por el acceso carnal, y este implica penetración por alguna de las vías mencionadas en el artículo 361.

Respecto de la aplicación de las reglas de iter criminis al delito de violación con homicidio, el Tribunal adscribe expresamente a la postura que rechaza su aplicación, defendida por Oliver y Rodríguez. Sin embargo, se extrańa una mayor reflexión sobre este punto, toda vez que, en primer lugar, el Tribunal no reconoce que existe otra postura, la cual sí admite la aplicación de las reglas indicadas a este tipo de delitos, y en segundo lugar, cita cómo único argumento para defender su postura el de texto literal, el cual, a nuestro juicio, carece de la fuerza suficiente, por sí solo, para tomar por correcta dicha opción. Hubiese sido positivo el desarrollo de razones axiológicas o teleológicas adicionales al razonamiento de texto.

Sobre el significado de la expresión "con ocasión", discrepamos de la tesis expuesta por el Tribunal. En efecto, este entiende que el requisito consiste en que exista una proximidad temporal, pero además que el homicidio ocurra con ocasión de actos que impliquen ejecución o comienzo de ejecución de la violación. Según nuestra opinión, el estándar del Tribunal es excesivo, ya que identifica el requisito con una idea de identidad de acción, vale decir, que el mismo acto sea parte tanto de la ejecución del delito de violación como del ilícito de homicidio. Este estándar sería incluso superior al del concurso ideal del artículo 75. Estimamos que la postura correcta es la de Garrido, quien expresa que "con ocasión" significa que ambos ilícitos se hayan cometido en un mismo contexto fáctico, lo cual implica proximidad temporal.

Finalmente, en relación al problema sobre las consecuencias de un eventual concurso aparente entre un delito de violación en estado de ejecución imperfecto, y un delito de abuso sexual en estado de consumación, lamentablemente la discusión no estuvo presente en la sentencia. El Tribunal afirma sin más la existencia de un concurso entre una violación en carácter de tentativa con un homicidio consumado, sin embargo no repara en que la penalidad de la violación tentada es inferior a la del abuso sexual propio consumado. Luego, sin duda alguna las acciones realizadas por parte del imputado calificaron este último delito, por tanto podemos señalar que la ausencia de una reflexión al respecto no puede sino apreciarse como un déficit argumentativo de la resolución comentada. 


\section{BIBLIOGRAFÍA CITADA}

Balmaceda, Gustavo (2014) Manual de Derecho Penal, Parte Especial. Santiago: Editorial Librotecnia, $731 \mathrm{pp}$.

Bullemore, Vivian; Mackinnon, John (2005) Curso de Derecho Penal. Santiago: Editorial LexisNexis, T. III, 261 pp.

Carrasco, Edison (2007) "El problema del sujeto activo del delito de violación y sus posibles vacíos legales". Revista Ius et Praxis, Vol. 13, $\mathrm{N}^{\circ}$ 2, pp. 137-155. Disponible en: http://www.scielo.cl/pdf/iusetp/ v13n2/art07.pdf

Etcheberry, Alfredo (1998) Derecho Penal. Santiago: Editorial Jurídica de Chile, T. III, 490 pp.

Garrido, Mario (2010) Derecho Penal, Parte Especial. Santiago: Editorial Jurídica de Chile, T. III y IV.

Labatut, Gustavo (2000) Derecho Penal. Santiago: Editorial Jurídica de Chile, 263 pp.

Muñoz, Juan (1992) El delito de detención. Madrid: Trotta, 312 pp.

Mañalich, Juan Pablo (2004) "El secuestro como delito permanente frente al DL de Amnistías". Revista de Estudios de la Justicia, $\mathrm{N}^{\circ}$ 5, pp. 11-33. Disponible en: http://web.derecho.uchile.cl/cej/ recej/recej4/archivos/tentativa\%20y\%20desistimiento\%20_JPM_ corregido\%20_16_.pdf.

Oliver, Guillermo (2013) Delitos contra la Propiedad. Santiago: Thomson Reuters, $591 \mathrm{pp}$.

Politoff, Sergio; Matus, Jean Pierre; y Ramírez, María Cecilia (2006) Lecciones de Derecho Penal Chileno, Parte Especial, 2a Edición. Santiago: Editorial Jurídica de Chile, 691 pp.

Ramírez, María Cecilia (2005) "La frustración en delitos de mera actividad a la luz de determinadas sentencias". Revista de Derecho de la Pontificia Universidad Católica de Valparaiso, Vol. XXVI, pp. 133-141. Disponible en: http://www.rdpucv.cl/index.php/rderecho/ article/viewFile/570/538.

Rodríguez, Luis (2015) Delitos Sexuales. Santiago: Editorial Jurídica de Chile, 458 pp.

Rodríguez, Luis (2009) "Robo con Homicidio". Revista de Estudios de la Justicia, $\mathrm{N}^{\circ} 11$, pp. 131-151. Disponible en: http://web.derecho. uchile.cl/cej/rej11/RODRIGUEZ\%20COLLAO\%20_12_.pdf

Vivanco, Jaime (2009) El Delito de Robo con Homicidio. Santiago: Legal Publishing, $81 \mathrm{pp}$. 